\title{
Effect of form factors in fits to photoproduction data
}

\author{
R. M. Davidson* \\ Department of Physics, Applied Science and Astronomy \\ Rensselaer Polytechnic Institute, Troy, New York 12180-3590 \\ Ron Workman ${ }^{\dagger}$ \\ Center for Nuclear Studies and Department of Physics \\ The George Washington University Washington, DC 20052
}

(October 29, 2018)

\begin{abstract}
We compare the effects of several form factor recipes in fits to pseudoscalar meson photoproduction data. The specific examples of pion and kaon photoproduction are used to illustrate how different choices can alter the results of such analyses.
\end{abstract}

PACS numbers: 25.20.Lj, 13.60.Le, 11.40.-q, 11.80.Cr

*davidr@rpi.edu

†rworkman@gwu.edu 
The study of meson photo- and electroproduction is enjoying a resurgence, due mainly to a program of precise measurements at new facilities in the U.S. and Europe. One important goal of these studies is an improved understanding of the baryon resonances and their photodecay amplitudes. It is also hoped that these new experiments and subsequent analyses will reveal states not found in previous fits to pion induced and pion production reactions.

In order to obtain resonance information from cross sections and polarization measurements, one either performs a multipole analysis, followed by a separation of resonance/background contributions, or directly fits data in terms of a model explicitly containing resonance parameters. In the absence of complete experimental information, some theoretical input is always necessary. The use of a truncated partial-wave series, for example, simplifies the problem considerably, but requires a model for the high partial waves. In the resonance region, these high partial waves have generally been taken from a Born approximation.

In some cases, particularly in pion photoproduction, a Born approximation can be justified, at least qualitatively, through comparisons with low-energy multipole amplitudes and cross sections. A number of low-energy s- and p-wave multipoles are remarkably similar to those generated using a (unitarized) Born approximation. The use of a Born approximation for higher partial waves also seems to account for the forward peaking seen in charged-pion production.

While the use of a Born approximation is helpful at low energies, serious problems arise as the energy increases. In particular, the use of a pseudovector coupling scheme in pion photoproduction is advantageous near threshold but diverges badly compared to data above the delta resonance region. In the Mainz analysis [四], this problem was handled by allowing the $\pi N N$ coupling to be a mix [2] of pseudoscalar and pseudovector terms, becoming purely pseudoscalar at higher energies. In the kaon photoproduction case, however, even the pseudoscalar scheme diverges over the region of interest, and several attempts have been made to introduce a cutoff scheme for Born contributions [3] [7].

In order to compare the different approaches, we write the charged pseudoscalar meson photoproduction amplitude off protons in terms of the usual four gauge invariant amplitudes,

$$
\epsilon \cdot M_{f i}=\bar{u}_{f} \sum_{j=1}^{4} A_{j} M_{j} u_{p}
$$

with the explicitly gauge invariant representation

$$
\begin{aligned}
& M_{1}=-\gamma_{5} \epsilon \cdot \gamma k \cdot \gamma \\
& M_{2}=2 \gamma_{5}\left(\epsilon \cdot p_{1} k \cdot p_{2}-\epsilon \cdot p_{2} k \cdot p_{1}\right), \\
& M_{3}=\gamma_{5}\left(\epsilon \cdot \gamma k \cdot p_{1}-\epsilon \cdot p_{1} k \cdot \gamma\right) \\
& M_{4}=\gamma_{5}\left(\epsilon \cdot \gamma k \cdot p_{2}-\epsilon \cdot p_{2} k \cdot \gamma\right)
\end{aligned}
$$

where $k$ and $q$ give the photon and pion four-momenta, and $p_{1}$ and $p_{2}$ are the respective initial and final baryon four-momenta. The pseudoscalar Born terms are given by

$$
A_{1}=\frac{G e F\left(s, m_{f}^{2}, \mu^{2}\right)}{s-m^{2}}\left(1+\kappa_{p}\right)+\frac{G e F\left(m^{2}, u, \mu^{2}\right)}{u-m_{f}^{2}} \kappa_{f},
$$




$$
\begin{aligned}
& A_{2}=\frac{2 G e}{\left(s-m^{2}\right)\left(t-\mu^{2}\right)} \hat{F}, \\
& A_{3}=\frac{G e F\left(s, m_{f}^{2}, \mu^{2}\right)}{s-m^{2}} \frac{\kappa_{p}}{m}, \\
& A_{4}=\frac{G e F\left(m^{2}, u, \mu^{2}\right)}{u-m_{f}^{2}} \frac{\kappa_{f}}{m},
\end{aligned}
$$

where $m$ is the mass of the proton, $\mu$ is the mass of the produced meson, $\kappa_{p}$ is the anomalous magnetic moment of the proton, $m_{f}$ is the mass of the final baryon, and $\kappa_{f}$ is the anomalous magnetic moment of the final baryon. For the strong coupling constant $G$, in the case of pion production, we take $G=\sqrt{2} G_{\pi^{0} p p}$ with $G_{\pi^{0} p p}^{2} /(4 \pi)=13.75$.

In eq. (3), $F(s, u, t)$ is a form factor modifying the $s-, u-$, or $t$-channel exchanges and the factor $\hat{F}$ is constructed to modify $A_{2}$ in a way such that any gauge-invariance-violating term can be cancelled by an additional contact term. The point-like Born terms are obtained by setting all form factors equal to unity. In the minimal substitution scheme of Ohta [ब, $\hat{F}=1$ and the $A_{2}$ amplitude is unmodified. In Ref. [6], the functional form of $\hat{F}$ was taken to be

$$
\hat{F}=a_{1} F_{1}(s)+a_{2} F_{2}(u)+a_{3} F_{3}(t),
$$

where

$$
\begin{aligned}
F_{1}(s) & =F\left(s, m_{f}^{2}, \mu^{2}\right), \\
F_{2}(u) & =F\left(m^{2}, u, \mu^{2}\right), \\
F_{3}(t) & =F\left(m^{2}, m_{f}^{2}, t\right),
\end{aligned}
$$

and $\hat{F}$ was subject only to the constraint $a_{1}+a_{2}+a_{3}=1$. In Ref. [7], it was shown that Haberzettl's form is unacceptable except at the soft photon point, and a much more restrictive form was found which is in all cases consistent with the pole structure of the amplitude. For charged-meson photoproduction, one allowed form for $\hat{F}$ is

$$
\hat{F}=F_{1}(s)+F_{3}(t)-F_{1}(s) F_{3}(t),
$$

which we will use to compare with previous works.

The Born terms can, in principle, give a significant contribution to charged-meson photoproduction, and their modification could alter the findings for associated resonance contributions. For example, in kaon photoproduction the divergence of a point-like-Born contribution to the total cross sections is well known, and attempts to cure this problem, by modifying the individual multipoles, were described in Ref. [3]. Ohta's prescription, while damping all but amplitude $A_{2}$, still gives a Born contribution which grows too rapidly. In Ref. [7] it was shown that the $\hat{F}$ form given in Eq. 6 successfully damps the Born contribution to the total cross section, and thus could be used in a phenomenological analysis of the photokaon data. It is difficult to judge how a proper treatment of $\hat{F}$ might affect the results of such an analysis, but we note there is a broad kinematic region where $\hat{F}$ is numerically close to $F_{3}(t)$ when we use standard expressions for the form factors; 


$$
\begin{aligned}
& F_{1}(s)=\frac{\Lambda^{4}}{\Lambda^{4}+\left(s-m^{2}\right)^{2}}, \\
& F_{2}(u)=\frac{\Lambda^{4}}{\Lambda^{4}+\left(u-m_{f}^{2}\right)^{2}}, \\
& F_{3}(t)=\frac{\Lambda^{4}}{\Lambda^{4}+\left(t-\mu^{2}\right)^{2}} .
\end{aligned}
$$

Thus, our $\hat{F}$ is numerically close to that used in Ref. [6], i.e., $\hat{F} \approx F_{3}(t)$ corresponding to a particular choice of their weight coefficients. However, this choice was not continued in subsequent works [8,9].

If form factors are important in the case of kaon production, one must consider their potential effect in pion production as well. This is of particular importance since most of our current knowledge of electromagnetic decays of nucleon resonances has been extracted from analyses of pion photoproduction data. Most of these analyses have used point-like Born terms which might bias the extracted resonance parameters. As in kaon production, the total cross section generated by the pseudoscalar Born terms for $\pi^{+} n$ production exceeds the experimental value starting at a photon lab-energy $\left(E_{\gamma}\right)$ of about $450 \mathrm{MeV}$. Putting in form factors, as above, is one way to cure this problem. However, the effect of these form factors on the differential cross section is cause for concern. The experimental cross section exhibits a forward peak which is usually associated with the Born terms. With the addition of form factors, the Born terms may lose this forward peaking behavior. The peak seen in forward $\pi^{+} n$ cross sections is a result of destructive interference amongst the small- $l$ multipoles and the large- $l$ multipoles generated by the $A_{2}$ amplitude in Eq. (3), which in a specific gauge can be interpreted as the $t$-channel pion exchange contribution. In many cases, the small- $l$ multipoles are fitted to the data while the large- $l$ waves are assumed to be given by the point-like Born terms. In other cases, the full point-like Born term is added to phenomenological resonance contributions. The effect of form factors in the fitting process is expected to be sensitive to this choice. Let us first consider how form factors modify the large- $l$ multipoles.

Note that large- $l$ multipoles are dominated by the $A_{2}$ amplitude and, ignoring irrelevant factors, to obtain the multipoles, one needs integrals of the form

$$
M_{l}=\frac{1}{2} \int_{-1}^{1} \frac{P_{l}(x)}{t-\mu^{2}}\left[F_{1}(s)+F_{3}(t)-F_{1}(s) F_{3}(t)\right] \mathrm{d} x
$$

where $x$ is the cosine of the center-of-momentum $(\mathrm{cm})$ scattering angle and Eq. 6 has been used for $\hat{F}$. Now define

$$
M_{l}^{0}=\frac{1}{2} \int_{-1}^{1} \frac{P_{l}(x)}{t-\mu^{2}} \mathrm{~d} x=-\frac{1}{2 q k} Q_{l}(z),
$$

with $z=\omega / q$ where $\omega(\mathrm{q})$ is the pion energy (three-momentum) in the cm frame. In the absence of form factors, $M_{l}^{0}$ would give the point-like result. Using our forms of $\hat{F}$ and $F_{3}(t)$, we obtain

$$
M_{l}=M_{l}^{0}-I_{l}\left(1-F_{1}(s)\right),
$$


where

$$
\begin{aligned}
I_{l} & =\frac{1}{2} \int_{-1}^{1} \frac{P_{l}(x)\left(t-\mu^{2}\right)}{\Lambda^{4}+\left(t-\mu^{2}\right)^{2}} \mathrm{~d} x, \\
& =-\frac{1}{2 q k} \operatorname{Re} Q_{l}\left(z_{\Lambda}\right)
\end{aligned}
$$

with

$$
z_{\Lambda}=\frac{\omega}{q}-\frac{i \Lambda^{2}}{2 q k}
$$

and $k$ is the energy of the photon in the cm frame.

To check the relative importance of $M_{l}^{0}$ and $I_{l}$, we make use of the series expansion in $1 / x$ for $Q_{l}(x)$, the leading term being proportional to $1 / x^{l+1}$. The usefulness of this series in evaluating $Q_{l}(x)$ depends on the particular value of $x$, but the important point to notice is that every term in the expansion for $Q_{l}\left(z_{\Lambda}\right)$ is suppressed by at least

$$
\left(\frac{z}{\left|z_{\Lambda}\right|}\right)^{l+1}
$$

compared to the same term in the expansion of $Q_{l}(z)$, where

$$
\left|z_{\Lambda}\right|=\sqrt{\frac{\omega^{2}}{q^{2}}+\frac{\Lambda^{4}}{4 q^{2} k^{2}}}>z .
$$

Thus, for sufficiently large $l, I_{l}$ is negligible compared to $M_{l}^{0}$. The $u$-channel can also contribute to the large- $l$ multipoles. However, this contribution is controlled by $Q_{l}\left(z_{n}\right)$ where $z_{n}=E_{f} / q, E_{f}$ being the final baryon energy in the $\mathrm{cm}$ frame. As $z_{n}>z$, one sees that the $u$-channel contribution is negligible compare to the $t$-channel contribution for sufficiently large $l$.

The usefulness of this argument depends on how large $l$ must be before $M_{l}^{0}$ dominates. This depends of the value of $\Lambda^{2} / 2 q k$ because when $s \rightarrow \infty, z_{\Lambda} \rightarrow z$. However, as most multipole analyses are restricted to $E_{\gamma}<2 \mathrm{GeV}$, we concentrate on energies in this range. For $l=0$ and 1 , form factors significantly affect the multipoles at almost all energies. For $l$ $=2$, form factor effects set in at about $E_{\gamma}=500 \mathrm{MeV}$, and with increasing $l$, form factors effects set in at higher $E_{\gamma}$.

Although the large $l$ multipoles are given very accurately by the Born terms without form factors, it could be the case that these large $l$ multipoles are unimportant. To check this, we show in Fig. 1 a comparison of the current SAID solution (solid line) with the experimental data [10] for differential cross sections at $E_{\gamma}=1002 \mathrm{MeV}$. To determine the importance of the $l \geq 6$ multipoles, we calculated the differential cross section using multipoles up to $l=$ 5 (dashed line). Evidently, the $l \geq 6$ multipoles are important in reproducing the forward peak. As a verification that the Born terms with form factors are capable of reproducing this peak, we have taken our amplitude for the Born terms with form factors, subtracted off their $l \leq 5$ multipoles, and added the rest to the SAID $l \leq 5$ multipoles. The resulting differential cross section is shown as the dotted line, which is nearly identical to the solid 
one. The full Born term (dashed-dotted) is also plotted with form factor modification, to show how the changes in lower partial waves affect the forward peaking behavior.

The results in Fig. 1 demonstrate that with a reasonable cutoff it is possible to damp the Born term contribution while at the same time not damp the high- $l$ multipoles which are needed to produce the forward peak in the cross section. While results will vary depending on what one chooses for the form factors, it is true in general that the high- $l$ multipoles will be dominated by the nearest singularity in $x$. This means that the high- $l$ multipoles will be given by the point-like-Born contribution unless one uses form factors which have a singularity in $x$ nearer than the one arising from the pion pole. Given these results, our conclusion is that multipole analyses of the type given in Ref. [11] are on firm ground in the sense that if Born terms with form factors were to be used in the analysis, the a priori unknown parameters in the $l \leq 5$ multipoles, which are fitted to data, would readjust to give a multipole solution very close to the present one. On the other hand, it is less clear how these form factors would influence results based on effective Lagrangian approaches [12] or isobar models [1]. If the analysis is restricted to the first resonance region, we expect no serious problem and, indeed, results for the Delta(1232) parameters obtained from various approaches are in good agreement [13]. However, if the analysis is performed over a larger energy region, problems may arise. At higher energies, the point-like-Born contribution must be partially cancelled by some mechanism, i.e., the resonance or vector meson exchange contributions, which could bias the extracted resonance parameters. We note that simply using Born terms with form factors is not the solution to this problem and might actually worsen the problem. If this were done, the small- $l$ multipoles, which are often resonance dominated, would have to readjust in order to produce the forward peak in the cross section, and thus, there is again the possibility of bias in the extracted resonance parameters.

\section{ACKNOWLEDGMENTS}

This work was supported in part by the U. S. Department of Energy Grants DE-FG0299ER41110 and DE-FG02-88ER40448. R.W. gratefully acknowledges a contract from Jefferson Lab under which this work was done. Jefferson Lab is operated by the Southeastern Universities Research Association under the U. S. Department of Energy Contract DEAC05-84ER40150. 
FIG. 1. Differential cross section for $\gamma p \rightarrow \pi^{+} n$ versus angle at $E_{\gamma}=1002 \mathrm{MeV}$. Plotted are the SAID [11] fit (solid), SAID fit minus waves beyond $l=5$ (dashed), and SAID fit with a

replacement of $l \geq 6$ waves with form-factor modified Born contribution (dotted). Also shown is the Born contribution with form factors (dashed-dotted). Data from Ref. [10].

\section{REFERENCES}

[1] D. Drechsel, O. Hanstein, S.S. Kamalov, and L. Tiator, Nucl. Phys. A645, 145 (1999).

[2] This approach is supported by the study of S. Kondratyuk and O. Scholten, Phys. Rev. C 59, 1070 (1999).

[3] H. Tanabe, M. Kohno, and C. Bennhold, Phys. Rev. C 39, 741 (1989).

[4] K. Ohta, Phys. Rev. C 40, 1335 (1989).

[5] R.L. Workman, H.W.L. Naus, and S.J. Pollock, Phys. Rev. C 45, 2511 (1992).

[6] H. Haberzettl, C. Bennhold, T. Mart, and T. Feuster, Phys. Rev. C 58, R40 (1998).

[7] R.M. Davidson and R.L. Workman, Phys. Rev. C, in press.

[8] T. Feuster and U. Mosel, Phys. Rev. C 59, 460 (1999).

[9] T. Mart and C. Bennhold, Phys. Rev. C 61, 012201(R) (1999); T. Mart, Phys. Rev. C 62, 038201 (2000); F.X. Lee, T. Mart, C. Bennhold, H. Haberzettl, L.E. Wright, nucl-th/9907119.

[10] Data from the SAID database (http://gwdac.phys.gwu.edu). This source also contains representative fits from several different groups.

[11] R.A. Arndt, I.I. Strakovsky, and R.L. Workman, Phys. Rev. C 53, 430 (1996).

[12] R.M. Davidson, N.C. Mukhopadhyay and R.S. Wittman, Phys. Rev. D 43, 71 (1991).

[13] R. M. Davidson, Nimai C. Mukhopadhyay, M. S. Pierce, R. A. Arndt I. I. Strakovsky and R. L. Workman, Phys. Rev. C 59, 1059 (1999). 


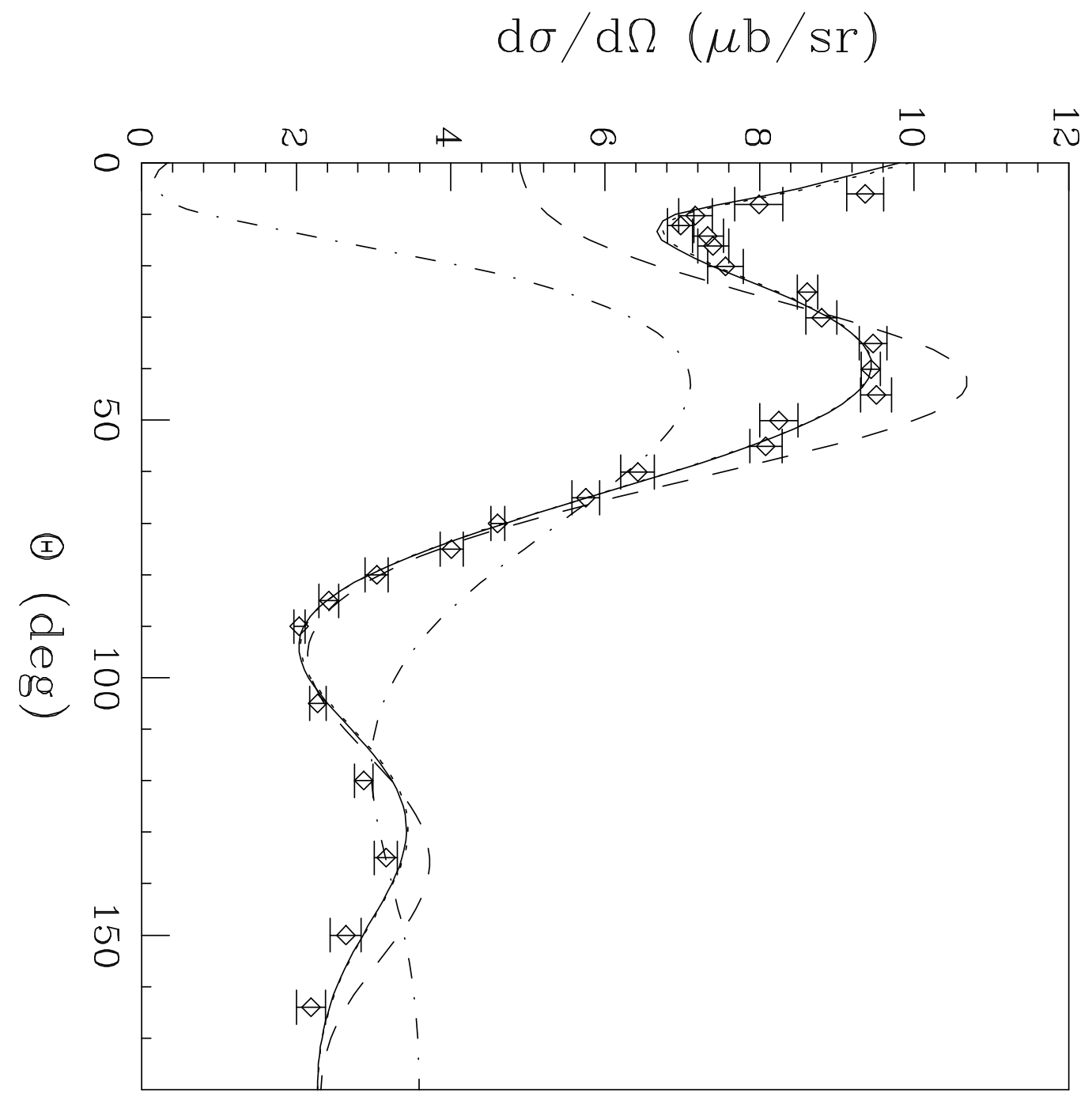

